\title{
Situación de la investigación educativo-musical en México. Retos, reflexiones y propuestas
}

\author{
The Situation of Music Education Research in Mexico. Challenges, Reflections, and Proposals
}

Situação da pesquisa educacional-musical no México. Desafios, reflexões e propostas

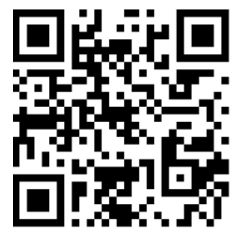

\author{
Raúl Wenceslao Capistrán-Gracia \\ Universidad Autónoma de Aguascalientes \\ Aguascalientes, México \\ raul.capistran@edu.uaa.mx \\ https://orcid.org/0000-0002-4594-8570
}

Recibido • Received • Recebido: 06 / 11 / 2019

Corregido • Revised • Revisado: 07 / 07 / 2021

Aceptado • Accepted • Aprovado: 12 / 08 / 2021

\begin{abstract}
Resumen:
Introducción. El mundo actual demanda de las instituciones de educación superior, personas investigadoras que generen conocimientos nuevos, promuevan su difusión y fomenten la vinculación, a fin de impulsar cambios y resolver problemáticas sociales. Objetivo. El objetivo de esta revisión bibliográfica consistió en determinar la situación actual de la investigación educativo-musical en México. Para lograrlo, se llevó a cabo una búsqueda documental sistemática, se localizaron fuentes primarias que proporcionaran información sobre el objeto de estudio en diversas bases de datos, repositorios de tesis y disertaciones, páginas web y textos. Metodología. La metodología utilizada fue el análisis de contenido, asistido por el programa ATLAS TI 8.0, por medio del cual se clasificó, estructuró y analizó la información reunida, se creó un esquema general y, a partir de él, se generaron las conclusiones. Resultados. Los resultados indican que ha habido algunos avances importantes, sin embargo, el número de personas investigadoras en educación musical es muy reducido, gran cantidad no está plenamente habilitado, no existen revistas nacionales especializadas en ese ámbito investigativo, la bibliografía del país es limitada y las reuniones académicas son escasas. Finalmente, el Padrón 2019 del Sistema Nacional de Investigadores indica que solo tres académicos tienen esa distinción. Conclusión. Existe un rezago importante, a pesar del impacto que la educación musical puede ejercer en el individuo. Se recomienda que las instituciones de educación superior implementen programas de formación de personal investigador para habilitarlo plenamente, y promueva sistemas de vinculación, para que los conocimientos generados tengan un uso inmediato en la sociedad.
\end{abstract}

Palabras claves: Investigación educativa; educación musical; educación superior; capacitación en investigación; vinculación académica. 
http://doi.org/10.15359/ree.25-3.30

http://www.una.ac.cr/educare

educare@una.ac.cr

\begin{abstract}
Introduction. Today's world demands from higher education institutions to have researchers that generate new knowledge, promote its diffusion, and foster linking programs with society in order to stimulate changes and contribute to the solution of its problems. Goal. The goal of this literature review was to determine the current situation of music education research in Mexico. A systematic documentary search was conducted to achieve this goal. Thus, primary sources providing information on the subject were located in various databases, repositories of theses and dissertations, web pages, and texts. Method. The methodology used was the analysis of content, assisted by the software ATLAS TI 8.0, through which the gathered information was classified, structured, and analyzed; a general scheme was created, and conclusions were generated. Results indicate there have been some important achievements. However, the number of researchers in music education is very small; many of them are not fully qualified; there are no national journals specialized in that field of research; bibliography by national authors is limited, and academic meetings are scarce. Finally, the 2019 Registry of the National System of Researchers indicates only three Mexican scholars have that distinction. Conclusion. Despite the impact that music education can have on the individual, there is an important lag in this field of research. Therefore, the author proposes that higher education institutions implement continuing education programs for researchers to support their full qualification and develop strategies to bridge the gap between research and society.
\end{abstract}

Keywords: Research in education; music education; tertiary education; research competencies; academic linking.

\title{
Resumo
}

Introdução. O mundo de hoje exige das instituições de ensino superior, pessoas pesquisadoras que gerem novos conhecimentos, promovam sua divulgação e incentivem o vínculo, a fim de promover mudanças e resolver problemas sociais. Objetivo. $O$ objetivo desta revisão de literatura foi determinar a situação atual da pesquisa na educação musical no México. Para isso, foi realizada uma busca sistemática de documentos, localizando-se fontes primárias para fornecer informações sobre o objeto de estudo em diversas bases de dados, repositórios de teses e dissertações, páginas da web e textos. Metodologia. A metodologia utilizada foi a análise de conteúdo, auxiliada pelo programa ATLAS TI 8.0, através do qual as informações coletadas foram classificadas, estruturadas e analisadas, um esquema geral foi criado e a partir daí, as conclusões foram geradas. Resultados. Os resultados indicam que houve alguns avanços importantes. Porém, o número de pesquisadores em educação musical é muito pequeno, muitos deles não são totalmente qualificados, não há periódicos nacionais especializados nesse campo de pesquisa, a bibliografia do país é limitada, e as reuniões acadêmicas são escassas. Finalmente, o registro de 2019 do Sistema Nacional de Pesquisadores indica que apenas três acadêmicos têm essa distinção. Conclusão. Há um atraso importante, apesar do impacto que a educação musical pode ter sobre o indivíduo. Como recomendação, as instituições de ensino superior devem implementar programas de formação para que pesquisadores sejam capacitados plenamente e promovam sistemas de vinculação, de forma que o conhecimento gerado tenha um uso imediato na sociedade.

Palavras-chave: Investigação educacional; educação musical; educação superior; treinamento em pesquisa; vinculação acadêmica. 


\section{Introducción}

Gradualmente, las instituciones de educación superior han demandado profesionales de la música, con niveles de maestría y doctorado, para atender los programas de licenciatura y posgrado. También, cubrir las funciones sustantivas de docencia, investigación, difusión y vinculación, y cumplir con los indicadores establecidos por organismos nacionales e internacionales (Barriga Monroy, 2009). Así, apenas a principios del siglo XXI, surgieron los primeros programas de posgrado en música en México (Universidad Veracruzana, 2017). Debido a diversas problemáticas de tipo académico, administrativo e inclusive laboral, aún se carece de profesorado con posgrado que estén habilitados como investigadores. De ese personal docente, solo un reducido número parece interesarse en la investigación educativomusical, constituyendo uno de los factores más importantes para el consiguiente atraso en el desarrollo de ese ámbito. Aunado a lo anterior, solamente hay tres centros especializados y dedicados a la realización de la investigación musical. No existen revistas especializadas en ese ámbito investigativo y, con excepción de las tesis derivadas de los pocos programas de maestría y doctorado, la producción académica publicada por equipos investigadores mexicanos es bastante escasa (García Rodríguez, 2019). A través de la revisión bibliográfica, cuyos resultados se presentan en este artículo, el autor proporcionará un panorama de la situación actual de la investigación educativo-musical en México, determinará sus fortalezas, valorará los logros, identificará algunas de las áreas de oportunidad más importantes, reflexionará sobre los retos que su superación implica y hará algunas propuestas de mejora.

\section{Marco teórico}

De acuerdo con Subirats Bayego (2011), en las últimas décadas del siglo XX, el campo de la investigación musical se dividió en tres ámbitos: musicología histórica, musicología sistemática y musicología aplicada. Sería en el ámbito de la musicología sistemática que se incluirían los estudios de tipo pedagógico. En ese sentido, Michels (1977) afirmaba: "sólo pertenece a la musicología en un sentido teórico, se ocupa de problemas de la educación musical, sus objetivos y métodos en los ámbitos privado y escolar" (p. 13).

Al igual que en las demás ciencias y disciplinas, la investigación en educación musical abarca dos dimensiones: la investigación básica y la investigación aplicada. La primera incluye el abordaje de aspectos teóricos y epistemológicos sobre los que se pueden fundamentar estudios posteriores. Mientras, la segunda implica la obtención de resultados, con el objetivo de proponer cambios e innovaciones que promuevan una mejora de los procesos de enseñanza y aprendizaje musical.

Si bien, de acuerdo con Phelps (1980), las investigaciones educativo-musicales pueden ser de tipo histórico, experimental, descriptivo, filosófico, o estético; el vacío principal que el autor desea destacar es el que se refiere a la investigación relativa a la propia práctica educativa. Así 
http://doi.org/10.15359/ree.25-3.30

http://www.una.ac.cr/educare

educare@una.ac.cr

pues, incluye temas tan amplios como las estrategias de enseñanza y aprendizaje utilizadas en el aula, la determinación de objetivos claros y concisos, la selección de contenidos apropiados o la implementación de métodos de evaluación adecuados, por mencionar solo algunos, pues, como afirma Subirats Bayego (2011, p. 189):

La investigación en el campo de la educación musical es reciente en los países latinos si se compara con los países anglosajones. Parece que cada vez más se investiga sobre la propia acción, al estilo del camino que marca la tradición anglosajona.

\section{Contextualización}

En México, la música universitaria nació en 1929, año en el cual se fundó la Facultad de Música de la UNAM, (Aguirre Lora, 2006; Capistrán Gracia, 2015). De acuerdo con Estrada Velasco y Estrada Rodríguez (1984), en 1946, el Instituto Nacional de Bellas Artes (INBA) creó la Sección de Investigaciones Musicales (SIM). Por tanto, la investigación musical se institucionalizó. Sin embargo, el SIM se especializó en el estudio de temas de tipo etnomusicológico, y, prácticamente, excluyó otros ámbitos investigativo-musicales. En 1968, la UNAM ofreció las primeras licenciaturas en música con reconocimiento de validez oficial por la Secretaría de Educación Pública (SEP) a través de la Facultad de Música, que entonces había cambiado su nombre a Escuela Nacional de Música (Universidad Nacional Autónoma de México [UNAM], 2011).

En años recientes, en la República Mexicana han surgido facultades y escuelas de música a nivel superior que ofrecen licenciaturas. La Asociación Nacional de Universidades e Instituciones de Educación Superior (ANUIES) registra 199 programas en música en México, ofrecidos por 48 universidades o instituciones de enseñanza superior en 27 de los 32 estados que conforman el país.

Como consecuencia del aumento de personas egresadas, y con el propósito de cumplir con los estándares de modernización, innovación e internacionalización establecidos por modelos que tratan de emular el Proceso de Bolonia (1999), (como el Tuning América Latina y el 6X4) (Gacel-Ávila, 2011), se volvió necesario impulsar la educación musical e incrementar su calidad. Del mismo modo, la demanda de posgrados en música creció (Capistrán Gracia, 2015). Aunado a lo anterior, profesionales de la música que ya laboran en esas instituciones han buscado obtener grados académicos de maestría y doctorado como medio de superación académica y económica.

En México, los primeros posgrados fueron impulsados muy recientemente. En 2001, la Universidad Veracruzana incluyó, por primera vez en el país, una maestría en Música en su oferta educativa (Universidad Veracruzana, 2017). Asimismo, en el 2004, la Universidad Nacional Autónoma de México comenzó a ofrecer una maestría y, por primera vez en este país, un Doctorado en Música (UNAM, 2004), ambos programas con siete salidas terminales. De manera 
http://doi.org/10.15359/ree.25-3.30

más actual, el INBAL creó una Maestría en Interpretación de la Música Mexicana y un Doctorado en Artes, el cual incluye líneas de formación en el área de la música. Finalmente, en la esfera de la educación privada, el Centro Mexicano de Postgrado en Música, A. C., en Puebla, México, ofrece también un Posgrado en Música.

Por otro lado, muchas universidades ofrecen programas alternativos que, de alguna forma, proporcionan al estudiantado la preparación para que puedan incursionar en la investigación musical. Así, por ejemplo, la Universidad Autónoma de Aguascalientes tiene una Maestría en Arte; la Universidad de Guadalajara ofrece una Maestría en Educación y Expresión para las Artes, así como una Maestría en Etnomusicología; el INBAL un Doctorado en Artes; y la Universidad Autónoma de Baja California una Maestría en Artes. Del mismo modo, la Universidad de Guanajuato, la Michoacana de San Nicolás de Hidalgo, la de Guadalajara y la Autónoma de Aguascalientes cuentan con un Doctorado Interinstitucional en Arte y Cultura. Cuando las universidades no cuentan con posgrados relacionados con las artes, los interesados e interesadas que no pueden trasladarse a otras ciudades buscan alternativas. Entonces, posgrados en historia o en ciencias sociales para incursionar en investigaciones de tipo musicológico, o posgrados en investigación educativa para habilitarse en la investigación educativo-musical.

Las instituciones educativas demandan que sus docentes de tiempo completo posean posgrados, que puedan llevar a cabo investigaciones que generen conocimientos nuevos, y sean capaces de ingresar al Sistema Nacional de Investigadores (SNI) del Consejo Nacional de Ciencia y Tecnología (Conacyt). También, resulta prudente que tengan el perfil deseable que establece el Programa para el Desarrollo Profesional Docente para el Tipo Superior (PRODEP) de la Secretaría de Educación Pública (SEP), que sean capaces de integrar Cuerpos Académicos (CA) $y$, por supuesto, que puedan tomar parte en los núcleos académicos básicos de los posgrados $y$, así, puedan dirigir al estudiantado en su formación académica y en sus investigaciones.

Por todo lo anterior mencionado, la investigación se ha convertido en una de las funciones sustantivas de la vida universitaria. De modo que sus autoridades esperan, no solo que se incursione en estos procesos, sino también que el número de docentes investigadores se incremente y, que los que ya han ingresado al Sistema Nacional de Investigadores, lleguen a la categoría más alta de ese organismo y se mantengan ahí. Adicionalmente, la preocupación por cumplir con los indicadores establecidos por organismos nacionales, tal como la Secretaría de Educación Pública (SEP), la Asociación de Universidades e Instituciones de Educación Superior (ANUIES) y el Padrón Nacional de Posgrados de Calidad (PNPC), así como por organismos internacionales como la Asociación de Universidades Iberoamericanas de Posgrados (AUIP), ha hecho que algunas universidades se preocupen, incluso, por tener preparado el reemplazo del profesorado que pudiese jubilarse. 
http://doi.org/10.15359/ree.25-3.30

http://www.una.ac.cr/educare

educare@una.ac.cr

\section{Método}

El propósito de la revisión bibliográfica, cuyos resultados se comparten en este trabajo, fue conformar un panorama sobre la situación de la investigación educativo-musical en México para, finalmente, hacer algunas propuestas para impulsar su desarrollo. Con esa finalidad en mente, se llevó a cabo una búsqueda documental sistemática, la cual tuvo como objetivo localizar fuentes primarias y secundarias que proporcionaran información sobre:

a) Las fortalezas y logros alcanzados en el ámbito de la educación musical.

b) Las áreas de oportunidad más importantes.

Así pues, se consultaron las bases de datos de Redalyc, Ebsco Host, Latindex, Scielo y Dialnet, entre otras. También, se hicieron búsquedas en los repositorios de tesis y trabajos de grado de varias universidades iberoamericanas. Dada la naturaleza del tema, las páginas web de varios organismos públicos que rigen la vida educativa y la actividad investigativa del país se constituyeron en fuentes de información de gran importancia. Información adicional fue obtenida de algunos libros publicados por organismos de investigación e instituciones educativas.

Se localizaron 45 trabajos, sin embargo, se excluyeron 16 de ellos, puesto que no contribuían para determinar la situación de la investigación en educación musical en México (por ejemplo, trabajos sobre la historia de la educación musical en México o sobre los beneficios de la educación musical, entre otros), lo cual dejó un total de 29 fuentes de información. El número de fuentes empleadas para este trabajo es relativamente pequeño, debido a que, como se verá más adelante, la investigación en el ámbito educativo-musical en México es muy reciente.

La metodología utilizada fue el análisis de contenido. Con la ayuda del programa ATLAS TI 8.0, el investigador importó los textos en pdf, analizó la información en busca de datos que permitieran identificar las fortalezas y logros alcanzados en el ámbito de la educación musical y determinar las áreas de oportunidad más importantes. Además, codificó los datos relevantes y los categorizó para, posteriormente, relacionarlos con datos surgidos de otras fuentes, como las páginas web y los Anuarios de la Asociación Nacional de Universidades e Instituciones de Educación Superior (ANUIES), o el Padrón de Beneficiarios del Sistema Nacional de Investigadores (SNI), el cual está representado por listados en formato Excel, para así conformar el panorama de la situación actual de la investigación educativo-musical.

\section{Resultados}

Los resultados de la revisión bibliográfica se presentan a continuación en dos grandes apartados. El primero se denomina logros alcanzados; en él se presentan los avances más significativos en el ámbito de la investigación educativo-musical, sin dejar de señalar algunos 
http://doi.org/10.15359/ree.25-3.30

aspectos contextuales relevantes. El segundo se denomina Retos, reflexiones y propuestas; en él se exponen las áreas de oportunidad más importantes, se hacen algunas reflexiones y se presentan algunas propuestas para superarlas.

\section{Logros alcanzados}

De acuerdo con el Padrón de Beneficiarios del Sistema Nacional de Investigadores (Consejo Nacional de Ciencia y Tecnología [Conacyt], 2019), solo 21 profesores y profesoras en algún ámbito relacionado con la música han recibido el nombramiento de "Investigador Nacional". De esos 21, cinco son candidatos o candidatas a Investigador Nacional; nueve tienen el nivel I; cinco son nivel II, uno (el Dr. Julio Luis Estrada Velasco) se encuentra en el nivel III y no hay investigadores eméritos.

De los 21 investigadores e investigadoras, ocho llevan a cabo su labor en el área de la musicología histórica, cuatro parecen dedicarse a ese ámbito investigativo (la información en el padrón no es clara) y solo tres abordan la educación musical. El resto se especializa en aspectos muy generales (por ejemplo, en uno de los casos el padrón solo menciona como especialidad la palabra "música"), o bien, enfatizan temas muy específicos, por ejemplo; "Instrumentación y procesamiento de señales". El listado en Excel, publicado en la página web del Sistema Nacional de Investigadores de Conacyt (Conacyt, 2019), despierta algunas dudas, debido a que una parte del listado no muestra el campo, la disciplina y la subdisciplina de los investigadores. Del mismo modo, resulta importante mencionar que el sistema informático elaborado por Conacyt para la generación del "Curriculum Vitae Único" y el registro de solicitudes SNI, exhibe algunas fallas importantes que causan confusión al momento de llenar los formularios. Entre esas fallas se encuentra que, de manera predeterminada, el sistema da la opción de musicología a todas las personas investigadoras que incursionan en el ámbito musical. Por lo anterior, podría haber más docentes involucrados en la investigación educativo-musical. Sin embargo, tal y como se refleja en el padrón, si tomamos en cuenta que la población de México asciende a 119,938,473 habitantes (Instituto Nacional de Estadística, Geografía e Informática [INEGI], 2015), implica que hay aproximadamente un investigador SNI en el ámbito de la educación musical por cada $39,979,491$ personas.

Como puede verse en la Tabla 1, de las especialidades desarrolladas por los investigadores musicales del SNI, la musicología histórica parece ser la disciplina más abordada. El aparente énfasis en ese ámbito podría tener su origen en la ya centenaria tradición musicológica mexicana, la cual parece tener sus inicios en la obra de Alba Herrera y Ogazón (1885-1931) y su libro El Arte Musical en México, publicado en 1917, así como en la obra de otros musicólogos como Gabriel Saldívar (1909-1981); Jesús C. Romero (1893-1958); y el norteamericano Robert M. Stevenson (1916-2012), entre otros. 
http://doi.org/10.15359/ree.25-3.30

http://www.una.ac.cr/educare

educare@una.ac.cr

Tabla 1: Especialidades en investigación musical de profesorado en el SNI, 2019

\begin{tabular}{lc}
\hline \multicolumn{1}{c}{ Especialidad } & Número de investigadores \\
\hline Musicología & 10 \\
Educación musical & 3 \\
Historia de la música popular en México & 1 \\
Música & 1 \\
Investigación en música, danza y cultura & 1 \\
Exilios y migraciones de la música iberoamericana & 1 \\
Teoría de la creación musical (filosofía) & 1 \\
Hermenéutica de la música mexicana & 1 \\
Iconografía musical & 1 \\
Instrumentación y procesamiento de señales & 1 \\
\hline Total & 21 \\
\hline
\end{tabular}

Nota: Elaboración propia con información derivada del Padrón de Beneficiarios (Conacyt, 2019).

Por otro lado, la educación musical como ámbito investigativo, está apenas representada en el mencionado padrón por tres docentes. Por supuesto, existen más personas en México que abordan la investigación educativo-musical. Carbajal Vaca (2016), reporta cómo, en diversas partes de México, existen investigadores e investigadoras que han contribuido a la generación de conocimientos nuevos en materia de educación musical, aseveración que puede corroborarse con algunos artículos escritos por mexicanos y mexicanas en revistas internacionales de reconocido prestigio como LEEME, RIEM, RECIEM, ArtsEduca, Revista Educación, y otras similares. Sin embargo, o no han cumplido con los indicadores que establece el SNI para recibir la distinción de Investigador Nacional, o simplemente no están interesados en obtenerla. En cualquiera de los casos, no se debe olvidar que el número de profesorado especializado en educación musical en el padrón del SNI (aun con la incertidumbre de que pudieran ser más) representa un reflejo de la actividad investigativa en ese ámbito. Además, es un indicador muy importante para las instituciones de educación superior.

La poca representatividad en el ámbito de la educación musical también podría tener una estrecha relación con su incipiente historia, la cual, de acuerdo con Estrada Velasco y Estrada Rodríguez (1984), tuvo sus inicios en la década de los 80 y se vio reflejada por escasos trabajos 
http://doi.org/10.15359/ree.25-3.30

llevados a cabo por pocos investigadores. Cabe mencionar que durante la primera mitad del siglo XX, Alba Herrera y Ogazón, Luis Sandi y Otto Mayer-Serra, entre otros, abordaron el tema de la educación musical, sin embargo, lo hicieron desde una perspectiva histórica. Del mismo modo, se reconoce una importante cantidad de trabajos metodológicos, pero no representan aportaciones al conocimiento desde la perspectiva investigativa que a este trabajo interesa.

En 1974, se fundó el Centro Nacional de Investigación, Documentación e Información Musical (CENIDIM), en un esfuerzo por impulsar el estudio, conocimiento, rescate, conservación y divulgación de la música de México (Centro Nacional de Investigación, Documentación e Información Musical Carlos Chávez [Cenidim], 2019). La labor de este organismo abarca ocho líneas de investigación, incluyendo la pedagogía musical, la cual se encarga de estudiar "la pedagogía y la enseñanza de la música en México en diversos aspectos: educación formal e informal, niveles básico, medio y profesional, planes y programas de estudio, materiales didácticos, historia de la educación musical, etc." (CENIDIM, 2015, citado en Carbajal Vaca, 2016, p. 28). Sin embargo, el Catálogo de Publicaciones (CENIDIM, 2015) de ese organismo, incluye solamente tres obras de Susana Dultzin que se relacionan con la investigación educativomusical y, hasta el día de hoy, no contiene más aportaciones bibliográficas en ese ámbito, lo que podría reflejar la poca o nula actividad en esa línea de investigación. Esa misma área de oportunidad se manifiesta en la revista Heterofonía, publicación periódica del CENIDIM, en la que solo se pueden encontrar unos pocos artículos relacionados con la investigación educativo-musical. Finalmente, también se ha visto reflejada en el libro Cuarenta años de investigación musical en México a través del Cenidim (Bitrán Goren et al., 2016) en el que no hay contribución alguna en ese rubro desde la perspectiva que a este artículo interesa. Aunque estuviese editada por la Secretaría de Cultura, lo mismo podría afirmarse de la revista Pauta, Cuadernos de Teoría y Crítica Musical.

El esfuerzo más significativo está representado por los Cuadernos Interamericanos de Investigación en Educación Musical, publicación semestral de la entonces Escuela Nacional de Música y mérito del Dr. Luis Alfonso Estrada Rodríguez. De acuerdo con la plataforma de Latindex, el número 1 de esta revista vio la luz en 2001 y el último en 2007 (Latindex, 2019). Sin embargo, al analizar la autoría de los distintos artículos, destaca que solo una tercera parte, aproximadamente, fueron escritos por investigadores mexicanos. Por otro lado, tomando en cuenta la ventaja investigativa que llevan, aparece un número importante de artículos de investigadores argentinos y brasileños de reconocido prestigio, como Ana Lucía Frega y Sergio Luiz Ferreira Defigueiredo.

Muy recientemente, han podido observarse cambios importantes que presagian el rompimiento de la inercia que llevaba la investigación educativo-musical. Por un lado, el Dr. José Luis Navarro Solís, director interino de CENIDIM, entre 2018 y 2019, intentó impulsar la investigación en ese ámbito. Así también, la maestra Gabriela Pérez Acosta, trató de promover 
http://doi.org/10.15359/ree.25-3.30

http://www.una.ac.cr/educare

educare@una.ac.cr

el área de la cognición musical (Carbajal Vaca, 2016). Del mismo modo, destaca el Coloquio Educación Auditiva y la Teoría de la Música (Edautemus) que, desde hace seis años organiza el mencionado Dr. Estrada, a través de los Programas de Doctorado y Maestría en Música de la Universidad Nacional Autónoma de México (programas que el fundó), así como el Coloquio Internacional de Educación Musical a Nivel Superior que desde hace ocho años organiza el Cuerpo Académico (CA) “Educación y Conocimiento de la Música” de la Universidad Autónoma de Aguascalientes. Sin embargo, de acuerdo con Carbajal (comunicación personal, 7 de mayo de 2019), el cambio de dirección en la investigación educativo-musical aún no se ve reflejado en congresos tan importantes como el que organiza el Consejo Mexicano de Investigación Educativa (COMIE), en el que varias veces, dada la poca participación de especialistas, la lectura de su ponencia ha tenido que ser programada en mesas apenas relacionadas con la educación musical. Por otro lado, en el país, únicamente, están registradas dos redes PRODEP de investigación en ese ámbito (García Rodríguez, 2019). Finalmente, al revisar los contenidos de revistas en otros idiomas, como el International Journal of Music Education o el Journal of Research in Music Education, es muy raro encontrar artículos escritos por mexicanos.

\section{Retos, reflexiones y propuestas}

\section{La investigación educativo-musical como reclamo social y como demanda educativa}

De acuerdo con numerosas investigaciones, la educación musical ejerce múltiples beneficios en la formación del ser humano (Hallam, 2010), por lo que importantes organismos internacionales tales como el Fondo de las Naciones Unidas para la Infancia (UNICEF) y la Organización de las Naciones Unidas para la Educación, la Ciencia y la Cultura (UNESCO), han constituido la educación artística como un derecho inalienable. En relación con lo anterior, los gobiernos y organismos no gubernamentales de muchas naciones han dado un gran impulso a la educación musical, a través de numerosos programas, como medio para reconstruir el tejido social y rescatar a los niños y las niñas, así como los y las jóvenes en estado de vulnerabilidad de las calles. Desgraciadamente, en México, la falta de conocimiento sobre los procesos de enseñanzaaprendizaje musical ha provocado que funcionen con múltiples áreas de oportunidad (Capistrán Gracia y Reyes Sosa, 2019).

En el ámbito de la educación musical a nivel superior, si bien, los procesos de enseñanzaaprendizaje sobre métodos tradicionales sigue dando frutos, el siglo XXI demanda que la educación musical superior se adapte a los cambios sociales, artísticos, culturales, económicos, e incluso tecnológicos, que se suceden con constante rapidez. En ese sentido, los programas educativos deben tomar en cuenta ámbitos de acción novedosos; proponer diseños curriculares más creativos y flexibles y establecer nuevos perfiles de egreso y, por consiguiente, docentes mejor preparados y en constante actualización, algo que, para tener éxito, debe fundamentarse en la investigación (Díaz Gómez, 2012). 
http://doi.org/10.15359/ree.25-3.30

\section{Complejidad del ámbito educativo-musical}

La educación musical, como ámbito investigativo, implica un complejo entramado de disciplinas y ciencias. Filosofía, antropología, didáctica, pedagogía, psicología, anatomía, física y medicina son solo algunas de las disciplinas que entran en estrecha relación con la educación musical, y que implican un gran reto para el investigador, en su esfuerzo por generar un conocimiento nuevo que pueda resolver una problemática.

Así, un profesor o profesora que desee involucrarse en la investigación educativo-musical debe poseer una amplia cultura y una actitud indagadora que lo impulse a documentarse e informarse continuamente. En muchos casos, el investigador o investigadora deberá buscar la asesoría de un o una especialista que lo ayude a incursionar en aquellos campos y disciplinas que, para él o ella, sean desconocidas. Una vez que haya conformado un marco teórico sólido y un estado de la cuestión completo, el investigador o investigadora podrá determinar una metodología viable y pertinente para su estudio, que dé como resultado un conocimiento nuevo, válido y confiable (Ortiz Lefort, 2010).

Por otro lado, la diversidad y complejidad de las problemáticas y vacíos existentes en el ámbito de la educación musical, requiere de metodologías igualmente diversas y complejas para conducir exitosamente una investigación. Así, el profesor y la profesora deberán conocer, aprender y manejar enfoques cualitativos y cuantitativos que le permitan obtener datos e información, a partir de los cuales generar conocimientos nuevos. En muchas ocasiones, deberá diseñar metodologías mixtas que le permitan triangular la información, con el fin de asegurar la validez y confiabilidad de los resultados obtenidos. Por supuesto, ese vasto panorama se considera suficiente para generar cierta resistencia en cualquiera, cuyo principal objetivo fue, en sus inicios, ser un simple músico (Carbajal Vaca, 2017).

\section{Carencia de formación sólida}

A pesar de su adscripción universitaria, las carreras musicales no han podido sustraerse totalmente a la tradición conservatoriana decimonónica, y han enfatizado la ejecución musical y la composición. Personal docente y estudiantes continúan metodologías de largo aliento y, alienados de la realidad actual, en muchos casos, siguen promoviendo un aprendizaje musical mecánico arraigado en la escuela del siglo XIX. En ese contexto, desarrollar competencias universitarias no musicales se vuelve todo un reto, pues asignaturas relacionadas con la pedagogía musical y la investigación son constantemente cuestionadas, y el personal docente debe acudir a cuanto medio le es posible para motivar al estudiantado y convencerlo de la importancia que estas materias tienen en su formación profesional (Carbajal Vaca, 2017). Entre los argumentos más utilizados por el profesorado, se encuentra el hecho de que, un alto porcentaje de las personas egresadas de licenciatura en música realizan la docencia como fuente primaria o secundaria de ingresos (Guadarrama Olivera, 2013). 
http://doi.org/10.15359/ree.25-3.30

http://www.una.ac.cr/educare

educare@una.ac.cr

En ese sentido, Phelps (1980), pionero en investigación musical y referente importante en ese ámbito, afirmaba que, para el músico que ha sido entrenado en destrezas no verbales, la idea de llevar a cabo una investigación parece más bien extraña. Por tanto, es hasta que egresan de la licenciatura y buscan oportunidades laborales u opciones para seguir superándose, que el estudiantado se da cuenta que las competencias rechazadas tienen un tremendo valor (Carbajal Vaca, 2017). Entonces, al ingresar a una universidad para obtener una maestría que los habilite para abrirse paso en el sistema docente universitario, se enfrentan al desafío de llevar a cabo una investigación, sin poseer una formación académica sólida. Como afirmó Phelps (1980), debido a la usual preocupación por el desarrollo y mantenimiento de las destrezas de ejecución, los educadores, a menudo, no están familiarizados con las connotaciones filosóficas y pragmáticas de la investigación.

Así, en experiencia del autor de este trabajo, en algunos casos, el nivel de maestría se ha convertido en un nivel formativo en el que el estudiantado apenas comienza a adquirir competencias académicas, las cuales se tuvieron que haber adquirido en pregrado, o incluso antes (por ejemplo, redacción de textos académicos, rudimentos de investigación, etc.). Más aún, todavía en el posgrado existe una reticencia consciente o inconsciente hacia la investigación, por lo que, en algunas ocasiones, el personal docente debe ejercer acciones disciplinarias para que el estudiantado trabaje con rigor académico. Asimismo, que también se aleje de intenciones de producción artística y dirija sus esfuerzos hacia la generación de conocimientos nuevos, como ha sucedido en la Maestría en Arte que se imparte en la Universidad Autónoma de Aguascalientes, en la cual el estudiantado, en su mayoría creadores (personas actoras, artistas plásticos, músicos) de manera consciente o inconsciente, dirigen sus esfuerzos hacia la creación y evitan profundizar en los aspectos investigativos (metodología, marco teórico, estado de la cuestión, etc.).

Finalmente, el resultado no siempre es el óptimo. La experiencia ha demostrado que, en algunos de los casos, el nuevo maestro, maestra, doctor, doctora, obtiene el grado con la intención de ingresar en el sistema universitario o de lograr una mejor plaza por lo que, tan pronto recibe el título deja de lado la investigación (Lomelí Gutiérrez, 2016). En el mejor de los casos, suele culminar su posgrado con un gran vacío en conocimientos habilidades y destrezas para seguir adelante en su labor (Ortiz Lefort, 2010). Por otro lado, el personal docente de tiempo completo que ha obtenido su maestría o doctorado en la modalidad profesionalizante, igualmente carecen de formación como investigadores. Por lo tanto, es vital que las universidades promuevan una concientización por parte del profesorado de tiempo completo sobre la importancia de la investigación en la vida universitaria y, así, promover su capacitación y habilitación plena para el desempeño de sus funciones. 
http://doi.org/10.15359/ree.25-3.30

\section{Falta de asesoría o mentoría para equipos investigadores neófitos}

Como se explicó en el apartado anterior, lograr uno o dos posgrados no habilita totalmente al investigador o investigadora, sobre todo si sus grados fueron obtenidos en una modalidad profesionalizante. De esta manera, siempre quedan vacíos formativos que deben ser llenados (Ortiz Lefort, 2010). En ese sentido, algunas universidades no parecen darse cuenta de esta área de oportunidad, y carecen de programas de capacitación o sistemas de asesoría o mentoría para investigadores e investigadoras, principalmente para aquellos que tienen poca experiencia.

Si bien, se espera que los cuerpos académicos subsanen esta deficiencia a través del trabajo colegiado, son muchas las personas investigadoras que no pertenecen a uno de ellos. Por otro lado, muchas veces no existen cursos específicos que ayuden a subsanar las áreas de oportunidad y, en muchos casos, los cursos que se ofrecen no contribuyen directamente para que la persona investigadora desarrolle adecuadamente el proyecto que está llevando a cabo (Ortiz Lefort, 2010). Por dar un ejemplo, una investigación de corte cuantitativo en el ámbito educativo-musical puede no verse beneficiada por un curso de estadística, en el que la persona instructora recurre continuamente a ejercicios que están relacionados con la agronomía o con la medicina. De esta manera, después de múltiples "palos de ciego", el personal docente se equipa con conocimientos, habilidades y destrezas para llevar a cabo su labor. Mientras tanto, verá rechazados un buen número de artículos y capítulos de libro, con la consiguiente frustración y deseo de claudicar e, inclusive, con la amenaza de perder su trabajo. Esto último ha comenzado a suceder en instituciones como la Universidad Autónoma de Aguascalientes, la cual ha condicionado el otorgamiento de la plaza definitiva al profesorado que no logra ingresar al SNI en su año de prueba, como se evidencia en la Convocatoria 2019 a Concurso de Oposición PRODEP de la Universidad Autónoma de Aguascalientes (Universidad Autónoma de Aguascalientes, 2019).

Algunas veces, se busca llenar los vacíos a través de esfuerzos aislados. Por mencionar un ejemplo, el autor de este artículo, a través del Centro de las Artes y la Cultura de la Universidad Autónoma de Aguascalientes, organizó dos cursos intensivos de apoyo a quienes realizan investigación. Sin embargo, esto no resulta suficiente para el investigador o investigadora incipiente, quien requiere de una asesoría continua que le ayude a resolver las numerosas dudas que surgen a lo largo de un proyecto.

Por todo lo anterior, es crucial que las autoridades universitarias, al igual que procuran la impartición de cursos periódicos de formación docente, también propongan cursos deformación de investigación e implementen programas de mentoría que orienten a los investigadores e investigadoras con poca experiencia a llevar a cabo su labor, ya que, en diversas ocasiones, cuando el investigador o investigadora se acerca a un colega con experiencia en busca de ayuda no recibe su apoyo, debido simplemente a la falta de tiempo o, incluso, a celos profesionales, como aconteció en varias ocasiones al autor de este trabajo. 
http://doi.org/10.15359/ree.25-3.30

http://www.una.ac.cr/educare

educare@una.ac.cr

\section{Falta de vinculación}

Durante una charla, en una reunión social entre músicos, una experimentada profesora de piano comentó con cierto desprecio: "Yo no sé para que obtienen un doctorado, si lo único que hacen después es escribir en vez de tocar". El comentario fue inquietante, pues no solo revelaba el poco aprecio a la labor investigativa y a lo que esta pudiera contribuir a la educación y a la práctica musical, sino que también hay un desconocimiento de las diversas actividades que un investigador debe llevar a cabo para generar un conocimiento nuevo.

Más aun, el comentario reflejaba una problemática que, desde hace algún tiempo, ha sido identificada en algunos estudios: la falta de vinculación entre los resultados de investigación y su aplicación en la vida real (Bonilla Marín, 2015; Ortiz Lefort, 2010). Por ejemplo, un estudio exploratorio, llevado a cabo en seis departamentos universitarios de música en México, con el propósito de determinar el nivel de enseñanza y aprendizaje de estrategias de práctica efectiva por parte de 84 profesores de instrumento y 252 estudiantes de licenciatura en música con especialidad en ejecución, reveló que, la gran mayoría del alumnado, no conocía bases de datos como Ebsco Host, Jstor, Latindex, Scielo, Scopus, Sage Journals, y otras similares. Asimismo, no consultaban las revistas especializadas que se encuentran en ellas para guiar su práctica. También, los resultados del estudio indicaron que la mayoría del personal docente que participó en el estudio no aprovechaba la información derivada de estudios empíricos sobre estrategias de práctica efectiva (Capistrán Gracia, 2017). Así, no es de extrañar que Phelps (1980) afirmara que el personal docente de música a menudo es incapaz de seguir la lógica de una persona investigadora, por lo que frecuentemente, no aprovechan la investigación que puede proporcionar respuestas prácticas a sus problemáticas.

Por otro lado, quizá las personas investigadoras se han mantenido en su zona de confort al presentar los resultados de sus estudios en reuniones académicas y publicarlos en revistas científicas. Además, no se han involucrado de manera proactiva y efectiva en la divulgación y aplicación de esos conocimientos, como, por ejemplo, a través de talleres en contextos reales. Si bien resulta responsabilidad del personal docente vivir plenamente su vida laboral y mantenerse informados(as), también es verdad que, conociendo el atraso en materia educativo-musical de nuestro país (Capistrán Gracia, 2018a, 2018b), es responsabilidad de los investigadores divulgar más los resultados de sus trabajos y hacerlos accesibles a los destinatarios, para que cumplan con su misión de servir. En ese sentido Phelps (1980) afirmó: En realidad, hay innumerables problemas significativos en la educación musical que deben resolverse. ¿No sería más útil concentrarse primero en aquellos que son de interés inmediato y valor práctico para la profesión?

Las situaciones arriba mencionadas son un pálido reflejo del vacío que existe entre la investigación y la práctica, la cual responde a un área de oportunidad que debe ser atendida, no para justificar la labor investigativa, sino para que el conocimiento logrado cumpla más fácil 
y rápidamente con su función de contribuir a la solución de aquel problema para el cuál fue obtenido. Finalmente, quizá sea la falta de vinculación la que hace que, algunos colegas no acreditados como investigadores, vean con desdén la labor que estos llevan a cabo, y opinen de manera categórica que todos los esfuerzos que los investigadores e investigadoras hacen por cumplir con los indicadores institucionales representan una egoísta manera de promover su superación personal, misma que nada abona a la vida académica universitaria, como fue la opinión de varias personas miembros de la Comisión Ejecutiva del Centro de las Artes y la Cultura, en ocasión de una solicitud de apoyo económico presentada para llevar a cabo un coloquio sobre la relación existente entre el arte, la imagen y el sonido (Comunicación Personal, 19 de junio de 2019).

\section{Conclusiones}

En 1929, el eximio compositor mexicano Carlos Chávez afirmaba enfáticamente: "México no necesita doctores ni bachilleres en música; necesita buenos ejecutantes de banda, de orquesta, de ópera y de ballet, etc., así como profesores de instrucción musical media ..." (El Universal, 1929, citado en Aguirre Lora, 2006, p. 100). Esos tiempos han quedado en el pasado, y, hoy en día, las instituciones de educación superior en México demandan que sus docentes, además de contribuir en la formación de los futuros sujetos profesionales de la música, posean una educación integral, que sean capaces de involucrarse en la investigación con el propósito de generar conocimientos nuevos que resuelvan problemáticas sociales, impulsen cambios y fomenten mejoras; que difundan esos nuevos conocimientos y que promuevan su vinculación con la sociedad (Nielsen, 2009; Peñalba, 2017; Porta, 2014).

Más aun, se espera que cumplan con una serie de indicadores que reflejen el compromiso de la institución ante la sociedad, y ante organismos como la SEP, Conacyt, ANUIES, PNPC y AUIP, por mencionar solo algunos. Sin embargo, a pesar del impacto benéfico que la educación musical puede ejercer en el ser humano (Hallam, 2010), la revisión bibliográfica reportada en este artículo ha revelado que la investigación en ese ámbito es escasa, que la productividad científica es poca y que el número de investigadores reconocidos por el SNI está apenas representado por 3 profesores y que no existen publicaciones académicas periódicas que difundan los pocos conocimientos generados.

A pesar del preocupante panorama arriba expuesto, se han identificado algunos cambios que presagian un crecimiento en este ámbito investigativo. Para empezar, el número de Cuerpos Académicos (CA) que abordan la educación musical como una de sus Líneas de Generación y Aplicación del Conocimiento (LGAC) ha aumentado, y, con ello, se ha incrementado el interés por incursionar en su investigación. Así, hoy en día, se organizan más congresos, coloquios, seminarios y jornadas, lo que ha favorecido la difusión de trabajos de investigación, así como la vinculación entre los CA. Del mismo modo, algunas editoriales universitarias han decidido 
http://doi.org/10.15359/ree.25-3.30

http://www.una.ac.cr/educare

educare@una.ac.cr

apoyar el esfuerzo de sus profesores y profesoras, y han comenzado a publicar trabajos que han contribuido al incremento del pequeño corpus bibliográfico nacional que en materia de educación musical existe. Adicionalmente, esta reciente situación ha comenzado a despertar el interés del estudiantado de las licenciaturas en música, que gradualmente comienzan a sentirse atraídos por la educación musical como carrera profesional, y ha motivado al alumnado egresado a incursionar en el posgrado y desarrollar investigaciones relacionadas con esta disciplina.

Por supuesto, aún existe un largo camino por recorrer. La investigación educativa musical en México ha surgido muy recientemente, existen pocos posgrados a través de los cuales se ha impulsado su desarrollo y se requiere del apoyo por parte de las autoridades educativas, específicamente de las autoridades de las instituciones de educación superior, para que el profesorado interesado pueda habilitarse, afrontar plenamente los retos que se le presenten y continuar impulsando y promoviendo este importante rubro investigativo.

Finalmente, se considera crucial que las personas investigadoras estrechen la distancia entre sí y la sociedad, escuchen y conozcan sus necesidades, así también, que generen el conocimiento que contribuirá a la solución de problemáticas y, asimismo, lo dispongan al alcance de sus destinatarios. Resulta vital crear una sinergia que beneficie de igual manera a las personas investigadoras y a las personas usuarias de los conocimientos que, de esa labor, se deriven.

\section{Declaración de Material complementario}

Este artículo tiene disponible, como material complementario:

-La versión preprint del artículo en https://doi.org/10.5281/zenodo.4796376

\section{Referencias}

Aguirre Lora, M. E. (2006). La Escuela Nacional de Música de la UNAM (1929-1940): Compartir un proyecto. Perfiles Educativos, 28(111), 89-111. http://www.scielo.org.mx/scielo. php?script=sci arttext\&pid=S0185-26982006000100005

Barriga Monroy, M.L.(2009).La investigación en educación artística:Una guía para la presentación de proyectos de pregrado y postgrado. El Artista, (6), 154-163. https://dialnet.unirioja.es/ ejemplar/243126

Bitrán Goren, Y., Gómez, L. A. y Navarro, J. L. (Coords.). (2016). Cuarenta años de investigación musical en México a través del Cenidim. Centro Nacional de Investigación, Documentación e Información Musical Carlos Chávez. https://www.researchgate.net/ publication/307466816_Cuarenta_anos de_investigacion_musical_en_Mexico_a_ traves del Cenidim/link/57c5faba08ae0a6b0dc8da88/download 
Bonilla Marín, M. (Coord.). (2015). Diagnóstico del posgrado en México: Nacional. COMEPO. https:// www.google.com/url?sa=t\&rct=j\&q=\&esrc=s\&source=web\&cd=\&ved=2ahUKEwi7zeqXq dHxAhWGmmoFHdttB34QFnoECBoQAw\&url=https\%3A\%2F\%2Fwww.posgrado.unam. $\mathrm{mx} \% 2 \mathrm{Fsitios}$ interes\%2Fdocumentos\%2Fcomepo_regiones.pdf\&usg=AOvVaw3xvLZKPL 4IDEFfJ080OJSO

Capistrán Gracia, R. W. (2015). Reflexiones sobre la investigación musical en México. Aguardiente, 1(1). https://aguaardiente.uaa.mx/01/caj03.html

Capistrán Gracia, R. W. (2017). La práctica musical efectiva. Revisión de literatura, resultados de investigación y propuesta metodológica. UAA.

Capistrán Gracia, R. W. (2018a). Necesidades de formación docente de los maestros de educación artística a nivel secundaria del Instituto de Educación de Aguascalientes. ArtsEduca, 19, 10-31. https://doi.org/10.6035/Artseduca.2018.19.1

Capistrán-Gracia, R. W. (2018b). Reflexiones sobre la educación artística a nivel básico en Aguascalientes: Implicaciones para la educación superior. Revista Electrónica Educare, 22(2), 1-14. https://doi.org/10.15359/ree.22-2.19

Capistrán Gracia, R. W. y Reyes Sosa, D. (2019). Las orquestas infantiles y juveniles. Propósitos, logros, retos. Trabajo presentado en el IV Coloquio Aproximaciones Interpretativas Multidisciplinarias en Torno al Arte y la Cultura. Aguascalientes, Universidad Autónoma de Aguascalientes.

Carbajal Vaca, I. S. (2016). Transmutaciones curriculares: El panorama de la investigación en educación musical en México. En J. P. Correa Ortega, I. S. Carbajal Vaca, y R. R. Moreno Martínez (Comps.), Educación musical universitaria: Filosofía y estrategias curriculares (pp. 27-36). Editorial Universidad Autónoma de Aguascalientes. https://www.researchgate. net/publication/319914680 Educacion musical universitaria Filosofia y estrategias curriculares httpwwwuaamxdireccionesdgdveditorialdocseducacion musical universitariapdf/link/59c1b7d4a6fdcc69b92bc98c/download

Carbajal Vaca, I. S. (2017). Educación musical superior: El desarrollo de competencias profesionales en músicos universitarios. En Memoria Electrónica del Congreso Nacional de Investigación Educativa COMIE (pp. 1-12). COMIE. http://www.comie.org.mx/congreso/ memoriaelectronica/v14/doc/0081.pdf

Centro Nacional de Investigación, Documentación e Información Musical Carlos Chávez. (2015). Catálogo de publicaciones del Cenidim. Autor. https://cenidim.inba.gob.mx/ publicaciones-1/catalogo.html

Centro Nacional de Investigación, Documentación e Información Musical Carlos Chávez. (2019). ¿Quiénes somos? Autor. https://cenidim.inba.gob.mx/ 
http://doi.org/10.15359/ree.25-3.30

http://www.una.ac.cr/educare

educare@una.ac.cr

Consejo Nacional de Ciencia y Tecnología. (2019). Sistema Nacional de Investigadores. Padrón de Beneficiarios. Autor. https://www.conacyt.gob.mx/index.php/el-conacyt/sistemanacional-de-investigadores

Díaz Gómez, M. (2012). Educación musical: Investigamos, luego avanzamos. Revista Electrònica d'Investigació i Innovació Educativa i Socioeducativa, 3(2), 67-76. http://www.in.uib.cat/ pags/cat/vol3 num2.html

Estrada Velasco, J. L. y Estrada Rodríguez, L. A. (1984). La música y las instituciones en el México actual (1958-1980). En J. Estrada (Ed.), La música de México (Vol. 5, pp. 79-113). Instituto de Investigaciones Estéticas, UNAM.

Gacel-Ávila, J. (2011). Impacto del proceso de Bolonia en la educación superior de América latina. Revista de Universidad y Sociedad del Conocimiento (RUSC), 8(2), 123-134. https:// rusc.uoc.edu/rusc/es/index.php/rusc/article/view/v8n2-gacel.html

García Rodríguez, M. (2019). La comunidad científica de investigación en educación musical en México: Aproximaciones a su caracterización. Trabajo presentado en el $6^{\circ}$ Coloquio Nacional y $3^{\circ}$ Internacional, de Educación Musical a Nivel Superior. Aguascalientes, México.

Guadarrama Olivera, R. (2013). Mercado de trabajo y geografía de la música de concierto en México. Espacialidades. Revista de temas contemporáneos sobre lugares, política y cultura, 3(2), 192-216. https://www.redalyc.org/pdf/4195/419545120007.pdf

Hallam, S. (2010). The power of music: Its impact on the intellectual, social and personal development of children and young people. International Journal of Music Education, 28(3), 269-289. https://doi.org/10.1177/0255761410370658

Instituto Nacional de Estadística, Geografía e Informática. (2015). Encuesta Intercensal 2015. https://www.inegi.org.mx/app/buscador/default.html?q=Encuesta+intercensal+2015

Latindex. (2019). Catálogo v1.0 (2002 - 2017). Cuadernos interamericanos de Investigación en Educación Musical. https://www.latindex.org/latindex/ficha?folio=10059

Lomelí Gutiérrez, C. L. (2016). El perfil del docente en la universidad del siglo XXI. En I. J. VelascoAragón y M. Páez-Gutiérrez (Coords.), Los retos de la docencia ante las nuevas características de los estudiantes universitarios. Proceedings T-XI (pp. 67-77). ECORFAN. https://www. ecorfan.org/proceedings/CDU XI/PROCEEDING\%20TOMO\%2011.pdf.

Michels, U. (1977). Atlas de música I. Alianza Atlas.

Nielsen, F. V. (2009). What is the significance of research for music education in practice? On relations between the practice of and the scientific approach to music education. Philosophy of Music Education Review, 17(1), 22-40. https://doi.org/10.2979/PME.2009.17.1.22 
http://doi.org/10.15359/ree.25-3.30

Ortiz Lefort, V. (2010). Los procesos de formación y desarrollo de investigadores en la Universidad de Guadalajara. Una aproximación multidimensional. Universidad de Guadalajara. http:// www.publicaciones.cucsh.udg.mx/pperiod/cgraduados/pdf/sin/4 Los procesos de formacion_y desarrollo de investigadores en la_Universidad de_Guadalajara.pdf

Peñalba, A. (2017). La defensa de la educación musical desde las neurociencias. Revista Electrónica Complutense de Investigación en Educación Musical 14, 109-127. https://revistas. ucm.es/index.php/RECl/article/view/54814

Phelps, R. P. (1980). A guide to research in music education. Scarecrow Press.

Porta, A. (2014). Formación, investigación y difusión científica: Los grandes retos para el avance y reconocimiento de la educación musical. Boletín SEM-EE. 15-16. https://www. academia.edu/7894352/Formaci\%C3\%B3n investigaci\%C3\%B3n_y difusi\%C3\%B3n_ cient $\%$ C $3 \%$ ADfica los grandes retos para el avance y reconocimiento de la educaci\%C3\%B3n musical

Subirats Bayego, M. dels À. (2011). La Investigación en didáctica de la expresión musical. Educatio Siglo XXI, 29(1), 175-194. https://revistas.um.es/educatio/article/view/119931

Universidad Autónoma de Aguascalientes. (2019). Convocatoria 2019 a concurso de oposición PRODEP de la Universidad Autónoma de Aguascalientes. Autor. https://www.uaa.mx/portal/ wp-content/uploads/2019/11/oposicionNOV19.pdf

Universidad Nacional Autónoma de México. (2004). Programa de maestría y doctorado en música. Presentación del programa. Autor. http://www.posgrado.unam.mx/musica/div/ planEstudios2004/pdf/presentacion.pdf

Universidad Nacional Autónoma de México. (2011). Presentación del programa. Programa de maestría y doctorado. Autor. http://www.posgrado.unam.mx/musica/div/ planEstudios2011/pdf/Presentaci\%C3\%B3n.pdf

Universidad Veracruzana. (2017). Plan de estudios de maestría en música. Autor. 